\title{
Theorie der inneren Feldemission in kubischen Kristallen*
}

\author{
Von J. Homilius und W. Franz
}

Aus dem Institut für theoretische Physik der Universität Münster in Westfalen

(Z. Naturforschg. 9a, 205-210 [1954]; eingegangen am 11. Dezember 1953)

\begin{abstract}
In einer vorangehenden Untersuchung haben wir die räumliche Verallgemeinerung der Formel von $\mathrm{Z}$ en er für die innere Feldemission gegeben. Dabei ergab sich eine exponentielle Abhängigkeit von der Feldstärke genau in der Gestalt der ursprünglichen ZenerFormel. Wie Sh ockley und Mitarbb. zuerst angegeben haben, muß jedoch der Zenersche Exponent durch einen anderen ersetzt werden, in welchen nicht mehr die Gitterkonstante, sondern nur die Ionisierungsenergie des Materials und die effektive Masse der Elektronen eingeht. In der vorliegenden Arbeit wird diese Modifikation an den früher erhaltenen Formeln angebracht und sodann die Bedingung für Feldemissionsdurchschlag von kubisch flächenzentrierten und raumzentrierten Kristallen abgeleitet. Dabei ergibt sich eine mindestens 20-proz. Richtungsabhängigkeit der Durchschlagsfeldstärke. Die erhaltenen Formeln werden auf $\mathrm{PbS}, \mathrm{PbTe}$, Si und Ge angewandt.
\end{abstract}

$\mathrm{I}_{\mathrm{s}}^{\mathrm{n}}$ $\mathrm{n}$ der folgenden Untersuchung soll die Durchschlagsfestigkeit kubischer Kristalle berechnet werden. Dabei stützen wir uns auf die räumliche Verallgemeinerung der Zener-Formel, welche wir in einer vorangegangenen Arbeit ${ }^{1}$ angegeben haben, sowie auf die von Homilius ${ }^{2}$ berechneten Eigenfunktionen in Brillouinscher Näherung. Weiterhin ersetzen wir die Formel von Zener ${ }^{3}$ durch die von Shockley u. Mitarbb. ${ }^{4}$ angegebene. Dieser Übergang vollzieht sich (siehe dazu etwa auch Franz ${ }^{5}$ § 4) dadurch, daß man im Exponenten der ZenerFormel an Stelle der Größe $m a I^{2} / 4 \hbar^{2}$ setzt: $(\pi / 4)$ $\sqrt{2 m^{*}} I^{3 / 2} / \hbar$. Darin bedeutet $a$ den Abstand zweier benachbarter Netzebenen von der Art, welcher für die betrachtete Zonengrenzfläche verantwortlich ist, $I$ den kleinsten Betrag der Energielücke auf dieser Zonengrenzfläche und $m^{*}$ die zu der zugehörigen Stelle am unteren Rand des Leitungsbandes gehörige effektive Masse. Für die mittlere Durchlässigkeit einer Brillouin-Ebene (B-Ebene) erhalten wir dann gemäß [II, (15)] den folgenden Ausdruck

$D=\left(\frac{\pi}{3}\right)^{2} \exp \left\{-A I^{3 / 2} / F_{\perp}\right\} ; \quad A \equiv \frac{\pi}{4} \frac{\sqrt{2 m^{*}}}{\hbar e}$,

wenn wir von dem Einfluß der zu anderen Netzebenen gehörigen Reflexionen zunächst absehen $\left(F_{\perp}=\right.$ Komponente des angelegten Feldes senkrecht auf der Netzebene). Die Verfeinerung dieser Formel wird uns $\S 3$ bringen.

* Dritter Teilauszug aus der Dissertation von J. Homilius, Münster 1953.

1 J. Homilius u. W. Franz, Z. Naturforschg. 9 a, 5 [1954], im folgenden als II zitiert.

2 J. Homilius, Z. Naturforschg. 8 a, 432 [1953], im folgenden als I zitiert.
Zur Berechnung der Durchschlagsfestigkeit des Kristalls benutzen wir die von $\mathrm{Franz}^{5}$ angegebene Formel (126):

$$
F=\sqrt{\frac{2 \varrho \varkappa A T}{e b v w t^{2}}} ; \quad \Delta T \equiv T_{\mathrm{krit}}-T_{0} .
$$

Darin ist $T_{0}$ die Außentemperatur, $T_{\text {krit }}$ die Temperatur, bei der das Material zerstört wird, $\varrho$ die Dichte, $x$ die spezifische Wärme des Isolators, $v$ die Anzahl der Valenzelektronen pro Volumen, $b$ die Beweglichkeit der freien Elektronen, $t$ die für den Durchschlag verfügbare Zeit und schließlich $w$ die zeitliche Wahrscheinlichkeit für den Austritt eines Valenzelektrons durch innere Feldemission.

Als einfaches theoretisches Beispiel sei die Berechnung der Durchschlagsfestigkeit bei rein kubischem Gitter $\Gamma_{c}$ vorangestellt. Nach [II, (25)] ist die zeitliche Emissionswahrscheinlichkeit, wenn lediglich der Fourier-Koeffizient $V_{100}$ das Gitterpotential bestimmt, gegeben durch

$w=\frac{e F a}{h}\left(D_{1} \cos \alpha_{1}+D_{2} \cos \alpha_{2}+D_{3} \cos \alpha_{3}\right)$.

Darin sind die $D_{i}$ unter Übergang zu der Shockleyschen Formel nach Gl. (1) mit $I=2 V_{100}$

$$
D_{i}=\left(\frac{\pi}{3}\right)^{2} \exp \left\{-A I^{3 / 2} / F \cos \alpha_{i}\right\}
$$

$I$ ist jetzt die Energielücke in der Mitte der würfelförmigen Begrenzungsfläche der B-Zone. Wir wollen

${ }^{3}$ Cl. Zener, Proc. Roy. Soc. A 145, 523 [1934].

${ }^{4}$ K. McAfee, E. J. Ryder, W. Shockley u. M. Sparks, Phys. Rev. 83, 650 [1951].

${ }^{5}$ W. Franz, Erg. exakt. Naturw. XXVII, 1 [1953]. 
voraussetzen, da $\beta \alpha_{1}$ der kleinste unter den drei Winkeln $\alpha_{i}$ ist. Dann ist nach (4) $D_{1}$ die größte der drei Durchlässigkeiten, und wir lösen zweckmäßig
Gl. (2) nach Einsetzen von (3) nach der im Exponenten von $D_{1}$ enthaltenen Feldstärke $F$ auf. Dann ergibt sich

$F=A \frac{I^{3 / 2}}{\cos \alpha_{1}}\left\{\ln \left[\left(\frac{\pi}{3}\right)^{2} \frac{e^{2} F^{3} a b v t}{2 \varrho x h \Delta T}\right]+\ln \left[\sum_{k=1}^{3} \cos \alpha_{k} e^{-A\left(\frac{1}{\cos \alpha_{k}}-\frac{1}{\cos \alpha_{1}}\right) I^{3 / 2} / F}\right]\right\}^{-1}$.

Setzt man hierin die Zahlenwerte der universellen Konstanten ein, dann erhält man folgende numerische Formel

$F=\cdot 17,5 \frac{I^{3 / 2}}{\cos \alpha_{1}} \sqrt{\frac{m^{*}}{m}}\left\{28,7+\log _{10}\left(\frac{a b v}{\varrho \varkappa \Delta T} F^{3} t^{2}\right)+\log _{10}\left[\sum_{k=1}^{3} \cos \alpha_{k} e^{-40 \sqrt{m^{*} / m}\left(\frac{1}{\cos \alpha_{k}}-\frac{1}{\cos \alpha_{1}}\right) I^{3 / 2} / F}\right]\right\}^{-1}$.

Darin ist $a$ in $\AA$-Einheiten, $I$ in $\mathrm{eV}, F$ in $10^{3} \mathrm{~V} / \mathrm{cm}$, $x$ in cal $/ \mathrm{g}^{0} \mathrm{~K}, \varrho$ in $\mathrm{g} / \mathrm{cm}^{3}, v$ in $\AA^{-3}$ und $b$ in $\mathrm{cm}^{2} / \mathrm{V} \mathrm{sec}$ gemessen. Das Polardiagramm der Durchschlagsfeldstärke, welches sich ergibt, wenn man nur den vor der Klammer stehenden Faktor der Gln. (5) und (6) berücksichtigt, ist genau ein Würfel.

Speziell ergibt sich für die Richtungen [100], [110], [111] des Feldes das Verhältnis $1: \sqrt{2}: \sqrt{3}$, also

$$
F_{[100]}: F_{[110]}: F_{[111]}=1: 1,41: 1,73,
$$

wie dies schon von Franz ${ }^{6} 1939$ angegeben wurde. Durch die in der geschweiften Klammer noch enthaltenen Richtungscosinus ergibt sich eine Abweichung von der Würfelgestalt, bestehend in einer Abrundung der Kanten und der Ecken, welche nicht mehr als $7 \%$ beträgt, aber immerhin bemerkbar sein kann. Um ein Bild von den entstehenden Abrundungen $\mathrm{zu}$ erhalten, nehmen wir ein Material mit der Gitterkonstanten $a=3 \AA$, einer Energielücke $I=1 \mathrm{eV}$ und $v=1 / a^{3}$ an und setzen $m^{*} / m=$ $1, b=10^{2} \mathrm{~cm}^{2} / \mathrm{V} \mathrm{sec}, \varrho \varkappa \Delta T=10^{2} \mathrm{cal} / \mathrm{cm}^{3}, t=10^{-2}$ sec. Die Feldstärken ergeben sich in diesem Fall zu 0,$735 ; 1,015$ und 1,229 (in Einheiten $10^{6} \mathrm{~V} / \mathrm{cm}$ ) für die Richtungen [100], [110], [111]. Ihr Verhältnis ist mithin

$$
F_{[100]}: F_{[110]}: F_{[111]}=1: 1,38: 1,67 .
$$

Im folgenden wollen wir ähnlich die Durchschlagsfestigkeit für das flächenzentrierte und das raumzentrierte kubische Gitter $\Gamma_{c}^{\prime}$ und $\Gamma_{c}^{\prime \prime}$ berechnen, und zwar in Abhängigkeit von der kristallographischen Orientierung des Feldes, wobei wir uns wie oben auf die Richtungen [100], [110] und [111] beschränken wollen. Der Übersicht wegen treiben wir

\footnotetext{
${ }^{6}$ W. Franz, Z. Phys. 113, 607 [1939].
}

die Berechnung der kritischen Feldstärke in zwei Näherungsstufen voran: Wir berücksichtigen in Abschnitt 1 zunächst nur je eine Fourier-Komponente des Gitterpotentials pro Zonengrenzfläche. In Abschnitt 2 wird auf die Wechselwirkung mehrerer Koeffizienten eingegangen.

\section{Erste Näherung}

Die erste B-Zone des kubisch flächenzentrierten Gitters $\Gamma_{e}^{\prime}$ wird begrenzt durch die Würfelflächen $\pm k_{x_{i}}=2$ und die Oktaederflächen $\pm k_{x} \pm k_{y} \pm k_{z}$ $=3$. (Vgl. I, Fig. auf S. 440*). Für die Durchlässigkeit an diesen Flächen können wir Gl. (4) verwenden, wenn wir dort für die Ionisierungsenergie $I$ die Energielücke jeweils in der Mitte der betreffenden Grenzfläche der B-Zone nehmen, wofür wir in diesem Abschnitt nur den für diese Grenzfläche zuständigen Fourier-Koeffizienten heranziehen wollen; das bedeutet für die Würfelflächen: $I=I_{200} \equiv 2 V_{200}$, für die Oktaederflächen $I=I_{111} \equiv 2 V_{111}$. Für den Netzebenenabstand haben wir $a / 2$ bzw. $a / \sqrt{3}$ einzuführen, wenn wir jetzt unter $a$ den Abstand von einem Gitterpunkt bis zu dem in Richtung der $x$-Achse nächsten Nachbarn verstehen. Damit wird

$D_{\{111\}}=\left(\frac{\pi}{3}\right)^{2} \exp \left\{-A\left(I_{111}\right)^{3 / 2} / F_{\{111\}}\right\} ; \quad I_{111} \equiv 2 V_{111}$ und

$D_{\{200\}}=\left(\frac{\pi}{3}\right)^{2} \exp \left\{-A\left(I_{200}\right)^{3 / 2} / F_{x}\right\} ; I_{200} \equiv 2 V_{200}$.

Die Emissionswahrscheinlichkeit pro Zeiteinheit ist nach [II, (12)]

$$
w_{[x, y, z]}=\sum \gamma_{\mathrm{m}}^{[x, y, z]} D_{\mathrm{m}}^{[x, y, z]} .
$$

* Die Figuren (jedoch nicht die Unterschriften) der Abb. 6 und 8 in I sind versehentlich vertauscht. 
$\mathrm{Zu}$ summieren ist darin über sämtliche Grenzflächen der B-Zonen, welche durch den Vektor der elektrischen Kraft von innen her getroffen werden. Der Frequenzkoeffizient $v$ ist für jede dieser Flächen gegeben durch

$$
v=\frac{e F}{\hbar} \cos \alpha \frac{F l}{V},
$$

worin $\alpha$ der Winkel zwischen Feldrichtung und Grenzfläche ist, $F l$ der Flächeninhalt der Fläche und $V$ das gesamte Volumen der B-Zone. In Ein heiten $\pi / a$ ist dieses Volumen 32, der Flächeninhal ${ }^{-}$ der Würfelflächen 2 , der der Oktaederflächen $3 \sqrt{3}^{t}$

$$
\begin{array}{r}
w=\frac{e F a}{8 h}\left\{D_{\{200\}} \cos \alpha_{1}+D_{\{020\}} \cos \alpha_{2}+D_{\{002\}} \cos \alpha_{3}+\frac{3}{2}\left|\cos \alpha_{1}+\cos \alpha_{2}+\cos \alpha_{3}\right| D_{\{111\}}\right. \\
+\frac{3}{2}\left|\cos \alpha_{1}+\cos \alpha_{2}-\cos \alpha_{3}\right| D_{\{11 \overline{1}\}}+\frac{3}{2}\left|\cos \alpha_{1}-\cos \alpha_{2}+\cos \alpha_{3}\right| D_{\{1 \overline{1} 1\}} \\
+\frac{3}{2}\left|\cos \alpha_{1}-\cos \alpha_{2}-\cos \alpha_{3}\right| D_{\{1 \overline{1} \overline{1}\}}
\end{array}
$$

Damit ergibt sich speziell

$$
\begin{aligned}
& w_{[100]}=\frac{e F a}{8 h}\left(\frac{\pi}{3}\right)^{2}\left[\exp \left\{-A\left(I_{200}\right)^{3 / 2} / F\right\}+6 \exp \left\{-\sqrt{3} A\left(I_{111}\right)^{3 / 2} / F\right\}\right], \\
& w_{[110]}=\frac{e F a}{4 \sqrt{2} h}\left(\frac{\pi}{3}\right)^{2}\left[\exp \left\{-\sqrt{2} A\left(I_{200}\right)^{3 / 2} / F\right\}+3 \exp \left\{-\sqrt{6} A\left(I_{111}\right)^{3 / 2} / 2 F\right\}\right], \\
& w_{[111]}=\frac{e F a}{16 \sqrt{3} h}\left(\frac{\pi}{3}\right)^{2}\left[6 \exp \left\{-\sqrt{3} A\left(I_{200}\right)^{3 / 2} / F\right\}+9 \exp \left\{-A\left(I_{111}\right)^{3 / 2} / F\right\}+9 \exp \left\{-3 A\left(I_{111}\right)^{3 / 2} / F\right\}\right] .
\end{aligned}
$$

Ermitteln wir hieraus wie in Gl. (5) bzw. (6) die Richtungsabhängigkeit, dann ergibt sich analog zu Gl. (7) eine brauchbare Näherung, wenn man von den Exponentialfunktionen nur diejenigen mit dem kleinsten Exponenten beibehält und die Richtungsabhängigkeit des vor den Exponentialfunktionen stehenden Faktors $F$ vernachlässigt:

$F_{[100]}: F_{[110]}: F_{[111]}$

$=\operatorname{Min}\left(\delta^{3 / 2}, \sqrt{3}\right): \operatorname{Min}\left(\sqrt{2} \delta^{3 / 2}, \sqrt{3 / 2}\right): \operatorname{Min}\left(\sqrt{3} \delta^{3 / 2}, 1\right)$.

Darin ist $\delta=\left|V_{200} / V_{111}\right|$; diese Größe ist für Kristalle von Typ A 1 und B 1 aus theoretischen Gründen stets größer oder höchstens gleich 1. Deswegen wollen wir uns bei den späteren Betrachtungen immer auf $\delta \geq 1$ beschränken. Ist $1 \leq \delta \leq(3 / 2)^{1 / 3}=1,145$, dann ist die Richtungsabhängigkeit (15) am schwächsten; sie betrüge dann maximal $\sqrt{3} / \sqrt{2}$ (also etwa $22 \%$ ), ein Betrag, welcher der experimentellen Beobachtung gut zugänglich wäre.

Die B-Zone des raumzentrierten Gitters $\Gamma_{c}{ }^{\prime \prime}$ ist ein Rhombendodekaeder mit den Begrenzungsflächen $\pm k_{x_{i}} \pm k_{x_{i}+1}=2$ (vgl. I, Fig. auf S. 438). Das Volumen in Einheiten $\pi / a$ beträgt 16, der Flächeninhalt einer Rhombendodekaederfläche $2 \sqrt{2}$. Die Durchlässigkeit für eine dieser Flächen ist

$D_{\{110\}}=\left(\frac{\pi}{3}\right)^{2} \exp \left\{-A I^{3 / 2} / F_{\{110\}}\right\} ; I \equiv 2 V_{110}$, und die Austrittswahrscheinlichkeit berechnet man mittels (12) zu

$$
\begin{aligned}
& w=\frac{a e F}{4 h}\left\{\left|\cos \alpha_{2}+\cos \alpha_{3}\right| D_{\{011\}}\right. \\
& +\left|\cos \alpha_{2}-\cos \alpha_{3}\right| D_{\{01 \overline{1}\}} \\
& +\left|\cos \alpha_{1}+\cos \alpha_{3}\right| D_{\{101\}}+\left|\cos \alpha_{1}-\cos \alpha_{3}\right| D_{\{10 \overline{1}\}} \\
& \left.+\left|\cos \alpha_{1}+\cos \alpha_{2}\right| D_{\{110\}}+\left|\cos \alpha_{1}-\cos \alpha_{2}\right| D_{\{1 \overline{10}\}}\right\} \text {. }
\end{aligned}
$$

Hieraus folgt speziell

$$
\begin{aligned}
& w_{[100]}=\frac{e F a}{h}\left(\frac{\pi}{3}\right)^{2} \exp \left\{-\sqrt{2} A I^{3 / 2} / F\right\}, \\
& w_{[110]}=\frac{e F a}{2 \sqrt{2} h}\left(\frac{\pi}{3}\right)^{2}\left[\exp \left\{-A I^{3 / 2} / F\right\}\right. \\
& \left.\quad+2 \exp \left\{-2 A I^{3 / 2} / F\right\}\right], \\
& w_{[110]}=\frac{\sqrt{3} e F a}{4 h}\left(\frac{\pi}{3}\right)^{2} \exp \left\{-\sqrt{6} A I^{3 / 2} / 2 F\right\} .
\end{aligned}
$$

Für die Richtungsabhängigkeit erhalten wir mit dem gleichen Grad der Näherung wie in Gl. (15)

$$
F_{[100]}: F_{[110]}: F_{[111]}=\sqrt{2}: 1: \sqrt{3 / 2} .
$$

Die Anisotropie beträgt damit rund $40 \%$.

\section{Zweite Näherung}

Um für das fä̈chenzentrierte Gitter $\Gamma_{c}^{\prime}$ die Feldemission genauer zu berechnen, müssen wir zunächst die mittlere Durchlässigkeit der Grenzflä- 
chen der B-Zonen bestimmen. Aus I, Tab. 2 entnehmen wir für die wichtigsten Punkte der Fläche $\{111\}$ die folgenden Werte für die Energielücke:

$$
\begin{gathered}
P_{3}=(111): I_{111}, \quad P_{6}=(210): 2 I_{111} \operatorname{Min}\left(1 ; \frac{1}{2}+\frac{\delta}{2}\right), \\
P_{5}=\left(2 \frac{1}{2} \frac{1}{2}\right): I_{111} \sqrt{2+\delta^{2} / 4} \\
\cdot \operatorname{Min}\left(1 ; \frac{1}{2}+\frac{3 \delta}{2 V \delta^{2}+8}\right) . \quad(20)
\end{gathered}
$$

Nehmen wir wie oben an, daß $\delta=\left|V_{200}\right| V_{111} \mid \geq 1$ ist, dann gelten jeweils die ersten Größen in den Klammern; für $1 \leq \delta \leq 2 \sqrt{2}$ liegt die Energielücke in $P_{5}$ zwischen derjenigen von $P_{3}$ und $P_{6}$. Für ein plausibles $\delta$ wenig größer als 2 können wir die im Exponenten von (1) auftretende Größe $I^{3 / 2}$ einfach durch die folgende parabolische Abhängigkeit ausdrücken*:

$$
I^{3 / 2}(\varrho)=\left(I_{111}\right)^{3 / 2}\left[1+\left(\sqrt{2}-\frac{1}{2}\right) \varrho^{2}\right] ;
$$

$\varrho$ ist darin der Abstand von $P_{3}$ in Einheiten $\pi / a$. In diesen Einheiten ist $\varrho_{6}=\sqrt{2}, \varrho_{5}=\sqrt{3 / 2}$. Für die mittlere Durchlässigkeit der Fläche $\{111\}$ ergibt sich

$\bar{D}_{\{111\}}=\frac{2 \pi}{3 \sqrt{3}}\left(\frac{\pi}{3}\right)^{2} \int_{0}^{\varrho_{6}} e^{-\left(1+(\sqrt{2}-1 / 2) \varrho^{2}\right) \mathrm{B}_{3}} \varrho \mathrm{d} \varrho$

mit

$$
B_{i} \equiv A I_{i}^{3 / 2} / F_{\perp} .
$$

Da für alle in Betracht kommenden Felder $B \gg 1$ ist, wird (22) näherungsweise

$$
\bar{D}_{\{111\}}=\left(\frac{\pi}{3}\right)^{2} \frac{1}{(2 \sqrt{2}-1) B_{3}} \frac{2 \pi}{3 \sqrt{3}} e^{-B_{3}} .
$$

Auf der Ebene $\{200\}$ betragen die wichtigsten Aufspaltungen

$$
\begin{aligned}
& P_{4}=(200): I_{200}, \quad P_{6}=(210): 2 I_{111}, \\
& P_{5}=\left(2 \frac{1}{2} \frac{1}{2}\right): I_{111} \sqrt{2+\delta^{2} / 4} .
\end{aligned}
$$

Die Durchlässigkeit der Fläche $\{200\}$ kann nur dann interessieren, wenn sie kleiner ist als die von $\{111\}$. In dem für $\{200\}$ günstigsten Falle eines Feldes in Richtung [100] beträgt das Verhältnis der Exponenten für $P_{4}$ und $P_{3}\left(V_{200}\right)^{3 / 2}: \sqrt{3}\left(V_{111}\right)^{3 / 2}$. Daher kann die Fläche $\{200\}$ nur dann bei irgendeiner Feldrichtung bevorzugt sein, wenn $\delta<\sqrt[3]{3}$. Für diesen Fall können wir aber in hinreichend guter Näherung $I^{3 / 2}$ durch eine Parabel darstellen:

$I^{3 / 2}(\varrho)=\left(I_{200}\right)^{3 / 2}+\left\{\left(2 I_{111}\right)^{3 / 2}-\left(I_{200}\right)^{3 / 2}\right\} \varrho^{2}$.

Für die mittlere Durchlässigkeit erhalten wir dies$\mathrm{mal} * *$

$$
\bar{D}_{(200)}=\left(\frac{\pi}{3}\right)^{2} \frac{\pi / 2}{(2 / \delta)^{3 / 2}-1} \frac{1}{B_{4}} e^{-B_{4}} .
$$

Um die Austrittswahrscheinlichkeit zu errechnen, hat man mit den gemittelten Durchlaßfaktoren (24) und (27) in (13) einzugehen. Die Gln. (14) erhalten dadurch zusätzliche Koeffizienten der einzelnen Exponentialfunktionen, deren Gestalt ohne weiteres ersichtlich ist, so daß wir darauf verzichten, sie explizit anzuschreiben. Aus ihnen erhält man die folgenden Formeln zur Bestimmung der Durchschlagsfeldstärke, wenn man nur die Exponentialfunktionen mit dem jeweils kleinsten Exponenten beibehält:

Feld $\|[100]:$

$F=17,5 \sqrt{\frac{m^{*}}{m}}\left(I_{200}\right)^{3 / 2}\left\{26,36+\log _{10}\left[\frac{a b v}{\varrho \varkappa \Delta T} \frac{m^{*}}{m} F^{4} t^{2}\right]-\log _{10}\left[\left([2 / \delta]^{3 / 2}-1\right)\left(I_{200}\right)^{3 / 2}\right]\right\}^{-1}$

$F=30,3 \sqrt{\frac{m^{*}}{m}}\left(I_{111}\right)^{3 / 2}\left\{26,51+\log _{10}\left[\frac{a b v}{\varrho \varkappa \Delta T} \frac{m^{*}}{m} F^{4} t^{2}\right]-\log _{10}\left[\left(I_{111}\right)^{3 / 2}\right]\right\}^{-1}$ für $I_{200}>3^{1 / 3} I_{111} ;$

Feld || [110]:

$F=21,4 \sqrt{\frac{m^{*}}{m}}\left(2 \mathrm{~V}_{111}\right)^{3 / 2}\left\{26,51+\log _{10}\left[\frac{a b v}{\varrho \varkappa \Delta T} \frac{m^{*}}{m} F^{4} t^{2}\right]-\log _{10}\left[\left(I_{111}\right)^{3 / 2}\right]\right\}^{-1} ;$

Feld $\|[111]$ :

$F=17,5 \sqrt{\frac{m^{*}}{m}}\left(2 V_{111}\right)^{3 / 2}\left\{26,34+\log _{10}\left[\frac{a b v}{\varrho \varkappa \Delta T} \frac{m^{*}}{m} F^{4} t^{2}\right]-\log _{10}\left[\left(I_{111}\right)^{3 / 2}\right]\right\}^{-1}$.

* Dieses Vorgehen wird durch die Untersuchungen in II gerechtfertigt.

** Im Gegensatz zu II wird hier $\bar{D}$ proportional zu $B^{-1}$. Dieser Unterschied rührt daher, daß wir jetzt $I^{3 / 2}$ in der Seitenmitte parabolisch angesetzt haben, wäh- rend in II Näherungsformeln benützt waren, nach welchen in der Seitenmitte eine (wenn auch geringfügige) Unstetigkeit in der Ableitung der Energiefläche vorliegt. 
Beim raumzentrierten Gitter $\Gamma_{c}{ }^{\prime \prime}$ hat man nach I, Tab. 2 die folgenden Energieaufspaltungen

$$
P_{2}=(110): I \equiv 2 V_{110}, \quad P_{3}=(111): 2 I, \quad P_{4}=(200): 3 I \operatorname{Min}\left(1 ; \frac{2}{3}+\frac{\mathrm{V}_{200}}{3 \mathrm{~V}_{110}}\right) .
$$

Wir können dies für Punkt $P_{2}$ und $P_{3}$ genau und für $P_{4}$ in hinreichender Näherung darstellen durch

$$
I^{3 / 2}(\varrho) \approx I^{3 / 2} \cdot\left(1+(2 \sqrt{2}-1) \varrho^{2}\right) .
$$

Die mittlere Durchlässigkeit der Fläche $\{110\}$ ergibt sich hieraus zu

$$
\bar{D}_{\{110\}}=\left(\frac{\pi}{3}\right)^{2} \frac{\pi}{2 \sqrt{2}} \frac{e^{-B_{2}}}{(2 \sqrt{2}-1) B_{2}} .
$$

Die Durchschlagsfeldstärke bestimmt sich nach (1) und (18) zu

$$
\begin{aligned}
& \text { Feld } \|[100]: \quad F=24,7 \sqrt{\frac{m^{*}}{m}} I^{3 / 2}\left\{26,69+\log _{10}\left[\frac{a b v}{\varrho \varkappa A T} \frac{m^{*}}{m} F^{4} t^{2}\right]-\log _{10}\left[I^{3 / 2}\right]\right\}^{-1}, \\
& \text { Feld } \|[110]: \quad F=17,5 \sqrt{\frac{m^{*}}{m}} I^{3 / 2}\left\{26,34+\log _{10}\left[\frac{a b v}{\varrho x \Delta T} \frac{m^{*}}{m} F^{4} t^{2}\right]-\log _{10}\left[I^{3 / 2}\right]\right\}^{-1}, \\
& \text { Feld } \|[111]: \quad F=21,4 \sqrt{\frac{m^{*}}{m}} I^{3 / 2}\left\{26,34+\log _{10}\left[\frac{a b v}{\varrho \varkappa \Delta T} \frac{m^{*}}{m} F^{4} t^{2}\right]-\log _{10}\left[I^{3 / 2}\right]\right\}^{-1} .
\end{aligned}
$$

\section{Anwendung auf spezielle Materialien}

Als Anwendungsbeispiele für dieTheorie der inneren Feldemission kommen nur Materialien mit geringer Energielücke in Frage, welche somit bei nicht zu tiefen Temperaturen elektronische Halbleiter sein müssen. Wir greifen zunächst $\mathrm{PbS}$ und $\mathrm{PbTe}$ heraus. Diese Halbleiter kristallisieren im Steinsalzgitter, sind somit Anwendungsbeispiele für $\Gamma_{c}{ }_{c}$. Die Energielücke ist für $\mathrm{PbS}$ nach Gibsen ${ }^{7} 1,17 \mathrm{eV}$, für $\mathrm{PbTe}$ nach $\mathrm{Putley}{ }^{8} 0,63 \mathrm{eV}$. Es ist nicht unplausibel, diese Werte mit $2 V_{111}$ zu identifizieren. Wir setzen zunächst in die Gln. (28) bis (31) für die dort auftretenden Materialkonstanten plausible Werte ein und erhalten dann folgende numerische Formeln:

$$
\begin{gathered}
F=17,5\left(I_{200}\right)^{3 / 2}\left\{22,38+4 \log _{10} F\right. \\
\left.-\log _{10}\left[\left([2 / \delta]^{3 / 2}-1\right)\left(I_{200}\right)^{3 / 2}\right]\right\}^{-1}, \\
F=30,3 I^{3 / 2}\left\{22,50+4 \log _{10} F-\log _{10} I^{3 / 2}\right\}^{-1}, \\
F=21,4 I^{3 / 2}\left\{22,50+4 \log _{10} F-\log _{10} I^{3 / 2}\right\}^{-1}, \\
F=17,5 I^{3 / 2}\left\{22,38+4 \log _{10} F-\log _{10} I^{2 / 2}\right\}^{-1} .
\end{gathered}
$$

Da uns das Verhältnis $I_{200} / I$ nicht bekannt ist, berechnen wir für die Richtung [100] aus (38) den minimalen Wert $(\delta=1)$ und aus (39) den maximalen. Dann erhalten wir für die beiden Materialien die folgenden Ergebnisse:

${ }^{7}$ A. F. Gibsen, Proc. Phys. Soc. B 64, 953 [1951].
$P b S: \quad F_{[100]}= \begin{cases}1,02(\min .), & F_{[110]}=1,20 ; \\ 1,65(\max .), & F_{[111]}=1,00 .\end{cases}$

PbTe: $F_{[100]}=\left\{\begin{array}{l}0,423 \text { (min.), } F_{[110]}=0,496 ; \\ 0,684(\max .), F_{[111]}=0,413 .\end{array}\right.$

Das Verhältnis der drei Feldstärken ergibt sich in beiden Fällen zu

$$
F_{[100]}: F_{[110]}: F_{[111]}=\left\{\begin{array}{c}
1,02 \\
1,65
\end{array}\right\}: 1,20: 1,00 .
$$

Hätten wir in den Gln. (38) bis (41) die geschweiften Klammern alle gleich groß genommen, dann ergäbe sich genau die Näherungsformel (15). Infolge der Unterschiedlichkeit dieser Klammern bekommen wir, wie der Vergleich zeigt, merkliche, aber nicht sehr bedeutende Korrekturen.

Als zweites Beispiel wollen wir die Halbleiter vom Diamanttyp, nämlich Silicium und Germanium, betrachten. Das Gitter besteht bekanntlich aus zwei flächenzentrierten Gittern, welche um 1/4 der Hauptdiagonale gegeneinander verschoben sind. Die Symmetrie-Eigenschaften dieser Anordnung haben zur Folge, daß sämtliche Fourier-Koeffizienten $V_{i k l}$ des Potentials ausfallen, deren Indices $i, k, l$ nicht sämtlich gerade oder sämtlich ungerade sind. Die Wellenzahlvektoren der vier Valenzelektronen pro Atom füllen das durch die Ebenen vom Typ $\{220\}$ eingeschlossene Rhombendodekaeder aus; dies ist gerade die erste B-Zone eines raumzentrierten Gitters von der halben Gitterkonstanten, welches wir

${ }^{8}$ E. H. Putley, Proc. Phys. Soc. B 65, 388 [1952]. 
in I, Abb. 8 dargestellt haben. Allerdings befinden sich bereits im Inneren dieses Dodekaeders die B-Ebenen $\{111\}$, deren Fourier-Koeffizient zwar nicht verschwindet, aber doch kleiner ist als der $\mathrm{zu}$ den Ebenen $\{220\}$ gehörige. Diese Ebenen schneiden die Dodekaederkanten sowohl in der Mitte als auch in der Umgebung der Punkte vom Typ (400) und bringen damit eine Modifizierung des Energielückenverlaufs von $\Gamma_{c}{ }^{\prime \prime}$ gemäß $(\mathrm{I}, 4)$. Wir wollen von diesen Einflüssen jetzt absehen und behalten uns vor, bei`späterer Gelegenheit darauf zurückzukommen, bzw. überhaupt die genaueren Eigenfunktionen anderer Autoren zu benützen (siehe etwa Her$\left.\operatorname{man}^{9,10}\right)$. Der Einfluß solcher Verfeinerungen auf die Durchschlagsformeln bleibt so lange geringfügig, als die Flächen der kleinsten Energielücke im Wellenzahlraum parallel zur Richtung [220] liegen.

Die Anwendung der Gln. (35), (36), (37) liefert die folgenden numerischen Formeln

$$
\begin{aligned}
& F=24,7 I^{3 / 2}\left(22,72+4 \log F-\log I^{3 / 2}\right)^{-1}, \\
& F=17,5 I^{3 / 2}\left(22,38+4 \log F-\log I^{2 / 2}\right)^{-1}, \\
& F=21,4 I^{3 / 2}\left(22,38+4 \log F-\log I^{3 / 2}\right)^{-1} .
\end{aligned}
$$

Die Energielücken betragen für Si $1,11 \mathrm{eV}$ und für Ge 0,76 eV. Damit erhält man

${ }^{9}$ F. Herman, Phys. Rev. 88, 1210 [1952]. für $S i$ :

$F_{[100]}=1,26 ; F_{[110]}=0,92 ; F_{[111]}=1,11 ;$

für $G e$ :

$F_{[100]}=0,68 ; F_{[110]}=0,50 ; F_{[111]}=0,60$.

Das Verhältnis ergibt sich in beiden Fällen zu

$$
F_{[100]}: F_{[110]}: F_{[111]}=1,36: 1,00: 1,20 .
$$

Auch hier ergeben sich gegenüber der Näherungsformel (19) merkliche, wenn auch nicht sehr bedeutende Korrekturen.

Die Absolutwerte der errechneten Durchschlagsfeldstärken sind vor allem deswegen mit einer gewissen Unsicherheit behaftet, weil die effektiven Massen der verschiedenen Materialien nur z.Tl. und nicht sehr genau bekannt sind. Bei unseren numerischen Beispielen wurde stets die effektive Masse gleich der Masse des freien Elektrons gesetzt. - Die Richtungsabhängigkeit der Durchschlagsfeldstärke auf der anderen Seite hängt ganz wesentlich von der Zonenstruktur des Wellenzahlraumes ab. Deshalb könnte man aus Messungen der Richtungsabhängigkeit Rückschlüsse auf die Zonenstruktur ziehen.

${ }^{10}$ F. Herman u. J. Callaway, Phys. Rev. 89, 518 [1953]

\title{
Die spezifische Wärme des flüssigen Schwefels
}

\begin{abstract}
Von H. Braune und O. Möller
Aus dem Institut für Physikalische Chemie der Technischen Hochschule Hannover (Z. Naturforschg. 9a, 210-217 [1954]; eingegangen am 2. November 1953)

Mit Hilfe eines adiabatischen Kalorimeters wurden erstmals die wahren spezifischen Wärmen des flüssigen Schwefels von 120 bis $420^{\circ} \mathrm{C}$ gemessen; aus den Ergebnissen wurden die Wärmeinhalte berechnet, die mit früheren Angaben verglichen wurden. Der Temperaturverlauf der spezifischen Wärme läßt sich mit großer Wahrscheinlichkeit nicht auf Grund der Annahme eines Gleichgewichts $S_{\lambda} \rightleftarrows S_{\mu}$ deuten, sondern man wird wohl Gebrauch machen müssen von der durch den Viskositätsverlauf nahegelegten Hypothese, daß im flüssigen Schwefel Polymerisation eintritt, wobei Menge und Länge der Ketten eine Funktion der Temperatur sind.
\end{abstract}

$\mathrm{D}$ ie spezifische Wärme des flüssigen Schwefels, die sowohl für thermodynamische Rechnungen wie für die Beurteilung der in ihm herrschenden inneren Gleichgewichte von Bedeutung ist, war bis-

1 C. C. Person, C. R. Acad. Sci., Paris 23, 162 [1846]; Ann. chim. phys. (3) 27, 252 [1849]; Ann. Phys., Lpz. 70, 300 [1847].

2 J. Classen, Z. Instrumentenkunde 11, 301 [1891]. her nur recht ungenau bekannt. Einige ältere, z. Tl. stark voneinander abweichende Angaben über mittlere spezifische Wärmen liegen von Person ${ }^{1}$, Clas sen $^{2}$, Jitaka ${ }^{3}$ und Dussy ${ }^{4}$ vor. Lewis und

${ }^{3}$ J. Jitaka, Sci. Rep. Tôhoku Imp. Univ. Ser. I 88, 99 [1919].

4 J. Dussy, C. R. Acad. Sci. Paris 123, 305 [1896]. 